\title{
Pentagram Sustainability Model
}

Framing of a holistic sustainability dimension model for assessment of Neighbourhood-level Urban Communities (NLUCs) using a semi-automated systematic literature review

\author{
Sudeshna Kumar 1*, Anirban Santara2 and Haimanti Banerji1 \\ 1 Department of Architecture and Regional Planning, Indian Institute of Technology Kharagpur \\ 2 Google India Ph.D. Fellow, Department of Computer Science and Engineering, Indian \\ Institute of Technology Kharagpur, India University \\ * Corresponding Author, Email: rini.sudeshna@iitkgp.ac.in
}

Received: July 29, 2019; Accepted: December 16, 2019

Key words: dimensions of sustainability, sustainability assessment, neighbourhood level urban communities (NLUCs),

\begin{abstract}
This paper systematically reviews the current approaches of assessing sustainability of Neighbourhood-level Urban Communities (NLUCs) to identify existing dimensions of sustainability including missing and neglected ones. The authors have adopted a systematic literature review (SLR) approach to provide an overview of state-of-the-art literature in urban sustainability assessment and measurement. The novelty of the content analysis is that it has been accomplished using an automated process of keyword extraction from the systematically selected literature using a Python-implementation of the popular Rapid Automatic Keyword Extraction (RAKE) algorithm. Finally, the paper proposes a pentagram that successfully incorporates five dimensions of sustainability: environmental, economic, social, institutional and cultural, with an overarching component of innovation likely to have an impact on each due to technological advancements and future trends. The outcome of this model will be a basic framework within which indicators can be assigned for assessing the sustainability of the NLUCs.
\end{abstract}

INTRODUCTION

The concept of sustainability is very elusive (Kramers et al., 2013) and incorporates a plethora of meanings (Marshall \& Toffel, 2005) which has raised debate about the emergence of competing definitions (Hopwood, Mellor, \& O'Brien, 2005; Gibson, 2006) of sustainability and sustainable development since the publication of the Brundtland report "Our Common Future" (Komeily \& Srinivasan, 2015). But most of these definitions incessantly refer to the critical concepts of intragenerational and intergenerational equity which are featured in the Brundtland report (Barrett \& Grizzle, 1999); although researchers have not reached a consensus about what timescale should be considered for the applicability of such concepts (Bond, Morrison-Saunders, \& Pope, 2012). This all ever-embracing concept needs to be decomposed and reconstructed into different dimensions for detailed study and practice (Wang, 2014). Subsequently, after the publication of the Brundtland report, sustainability has been majorly 
conceived in three dimensions, namely environmental, economic, and social (Hassan \& Lee, 2015; Huang, Wu, \& Yan, 2015; Tanguay et al., 2010; Elkington, 1997). In the past decade, within the broader context of implementing sustainability principles, the practice of assessing sustainability has emerged in varied forms (Bond, Morrison-Saunders, \& Pope, 2012) and has been embracing an ever-evolving range of processes (Pope et al., 2017).

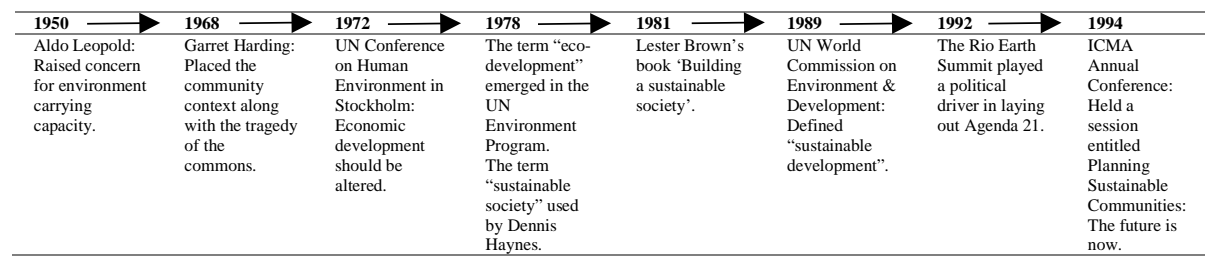

Figure 1. Timeline showing the evolution of the concept of sustainability

This is evident in the published book 'Sustainability Appraisal: A sourcebook and reference guide to international experience' (Dalal-Clayton \& Sadler, 2014). The 1994 ICMA Annual conference held a session titled "Planning Sustainable Communities: The Future is now" (refer Figure 1) which provided an international platform for discussing that sustainable communities is vital for optimising our future (Geis \& Kutzmark, 1998).

Sustainability assessment has been described as any process that aims to navigate decision-making towards sustainability and is considered to be the impact assessment tool of the new generation (Bond, Morrison-Saunders, \& Pope, 2012; Sharifi \& Murayama, 2015; Hacking \& Guthrie, 2008; Pope et al., 2017). In most instances, the process of sustainability assessment is different from the traditional process of impact assessment and is posed as a process 'explaining sustainable solutions to persistent problems'. The existing impact assessment tools adopt sustainability as a means to structure a policy that will be developed and evaluated to deliver alternative strategies to address problems (Pope et al., 2017). Wang (2014) review on evaluation methods of sustainability has suggested that the indicator method is the most widely used adopted method for assessment of sustainability development.

\subsection{Need for sustainability assessment of Neighbourhood-level Urban Communities (NLUCs)}

The authors opine that the neighbourhood scale is important in keeping alive the feeling of a community since most people perform their daily activities within this physical space. Within the wider spectrum of cities, it is at the neighbourhood scale where individuals, families and groups identify and experience a sense of belonging and there is scope for bringing about positive change. It is at the neighbourhood level where local residents and users of that physical and social environment can actively contribute to putting change into effect (Bijoux \& Pathway, 2012). Community refers to the group of people residing within a neighbourhood, whereas an urban neighbourhood as described by HQE2R is an urban (residential) area bounded by the large roads or other (linear) structures referring to the built environment which are more or less homogenous in character (Blum, 2007). In order to assess the sustainability of neighbourhoods, the authors felt the need to focus on the people rather than just the urban fabric and space since existing sustainable urban models/assessment tools fall short of an integrated approach towards sustainable development at the community level. Hence the authors have coined the term Neighbourhood-level Urban Communities 
(NLUCs) implying the neighbourhood in its totality referring to both the group of people residing within a tangible space with well-defined physical boundaries along with its natural and man-made fabric.

The study identifies the research gap for devising tailor made sustainability assessment tools for neighbourhood level urban communities. Most of the assessment tools used by the researchers referred to in the earlier section operated either at an urban and regional level or at the level of a single building, neglecting issues at the intermediate level of urban neighbourhoods (Blum, 2007). But, the turn of the $21^{\text {st }}$ century necessitates a need for assessing sustainability at the level of a neighbourhood, especially in developed countries (Sharifi \& Murayama, 2013). There exists evidence of ample research conducted across the globe on identifying indices for ensuring sustainability at the neighbourhood level, but many of the existing tools have a bias towards environmental sustainability (Berardi, 2013) focusing mainly on ecological parameters of the cities, neighbourhood and communities (Haapio, 2012; Kyvelou et al., 2012) and environmental performance of single buildings (Bond, Morrison-Saunders, \& Pope, 2012; Sharifi \& Murayama, 2013, 2014). Recent literature has acknowledged the limitation of such tools operating at the single building level (Wackernagel \& Rees, 1998) and has emphasized going beyond the scale of single buildings and focuses on the need for assessment of sustainability at the neighbourhood level.

Planners and policymakers have understood the importance of the neighbourhood as the microcosm of cities (Searfoss, 2011) and it is at the neighbourhood level where local residents and users of that physical and social environment can actively contribute to putting the change into effect (Bijoux \& Pathway, 2012), and hence neighbourhood sustainability assessment is gaining attention in sustainable planning for rendering sustainable development in cities (Sharifi \& Murayama, 2015). There exists a research gap in quantifying the level of sustainability or quantifying the performance of a community over segregated/specialized neighbourhoods (Cheshire, 2007). Since $70 \%$ of the world population will be thriving in urban settings in the third decade of the $21^{\text {st }}$ century, several Neighbourhood Sustainability Assessment (NSA) tools are being developed to respond to the need of transitioning cities to more sustainable communities.

\subsection{Absence of a tailor-made NSA tool framework in India}

India is one of the most ethnically diverse nations in the world. Rapid urbanization in Indian mega cities has also resulted in the concoction of varied cultural values and traditions over the ages. India is culturally rich and diverse, where any policy or plan of the government cannot survive without taking regional specificities into consideration. In the age of globalization, cultural sustainability is an emerging issue which must be dealt with with utmost urgency in order to sustain the traditional and local practices while adopting modern global trends, and the same is particularly true for India. Global organizations such as the World Bank, UN-Habitat and Asian Development Bank have taken significant initiatives across different countries to measure sustainability. But these global initiatives have encountered problems in implementing their strategy in India because they have failed to address country specific constraints (Panda, Chakraborty, \& Misra, 2016). Hence it is very important to have a tailor made 
comprehensive sustainability framework to address the problems which are typical to India (Panda, Chakraborty, \& Misra, 2016).

The authors have investigated and found that all the existing sustainability assessment tools in India, such as LEED India, GRIHA and the IGBC Green Township rating system, cover mainly environmental criteria (Bhatt, Macwan, \& Bhatt, 2012). Most of these existing sustainability assessment frameworks are based on the precedents of developed countries, hence the indicators used in these frameworks are generalized and not region-specific and have failed to accommodate local characteristics (Lin \& Shih, 2016). These NSA tools have been incessantly improvised but have stated that they lack "context-specificity", and are subject to a "one-size-fitsall” vision (Komeily \& Srinivasan, 2016). Context specificity suggests that neighbourhood sustainability assessment should be a "pluralistic practice" which would acknowledge specific characteristics of the local context, consider the opinions of the local communities and include involvement of the residents in the process (Sharifi \& Murayama, 2015; Berardi, 2013; Bond, Morrison-Saunders, \& Pope, 2012). The authors have felt the need to devise a tailor-made NSA tool for India to measure the sustainability of NLUCs. The existing tools have references to certain partial aspects pertaining to economic, social and environmental dimensions, and culture has been neglected altogether with no regard to context specificities of Indian cities.

Besides identifying the lack of a context specific sustainability assessment framework in India, the authors have also recognized the need for exploring and analysing the existing models on sustainability, to identify any missing dimension. Due to the abundance of literature in this field, a need is felt to conduct a data driven systematic literature review (SLR) study in order to validate the missing dimensions from a large pool of literature.

The foremost aim of this paper is to devise a new holistic model specifically for NLUCs which would overcome the limitation in the existing models. The model will act as a framework for a tailor-made NSA tool for Indian mega cities. This paper aims to fill the research gap by presenting the methodology and results of a semi-automated SLR in the area of urban sustainability assessment of neighbourhoods from 1998 onwards. The content analysis of the literature review has required the authors to outline a holistic comprehensive framework for assessing the sustainability of neighbourhood level urban communities in India. The dimension model will provide a conceptual framework for holistically assessing the sustainability of NLUCs, which implies "a network, or a plane of interlinked concepts that together provide a comprehensive understanding of a phenomenon" (Jabareen, 2009). The model will assist urban planners and policymakers in formulating more pragmatic and focussed policies to improve the sustainability of NLUCs.

Section 2 of this paper explains the methodology of the semi-automated SLR conducted in the study. Section 3 presents the analysis and results of the SLR. Section 4 presents the discussion and proposes a novel pentagram sustainability dimension model followed by the concluding section. Section 5 represents the concluding remarks highlighting the application of the framework of the proposed dimension model. 


\section{MATERIALS AND METHODS}

This paper presents an SLR of urban neighbourhood sustainability assessment to (1) identify the existing dimensions of sustainability, (2) identify the missing and neglected dimensions of sustainability, and (3) present a holistic comprehensive sustainability dimension model.

The authors have strategically collected data from digital libraries, following up with data selection and quality assessment. Rapid Assessment Keyword Extraction (RAKE) algorithm has been adopted and an automated binary classifier has been devised for data extraction followed by data synthesis and analysis, resulting in the identification of missing and existing dimensions of sustainability. Sensitivity and specificity analyses were conducted for measuring the performance of a binary classifier. The entire workflow of the study has been depicted in Figure 2.

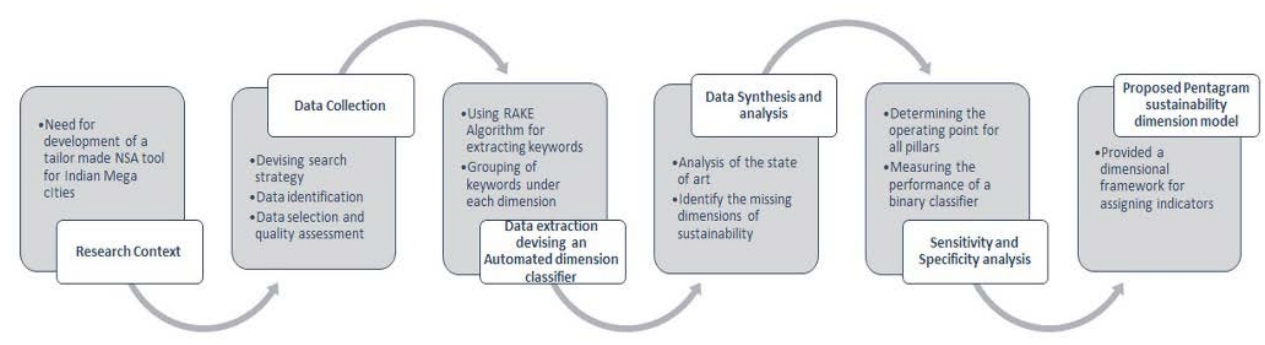

Figure 2. Showing the sequence of the workflow

This section describes the methodology adopted for conducting the SLR of urban neighbourhood sustainability assessment literature. The authors have adopted semi-automated literature methodology because there is abundant literature in the field of sustainability, with innumerable terminologies and overlapping research, hence automating the data extraction and analysis part would expedite the entire process of the literature review.

The main aim of a literature review is to analyse the current state or best practice of any particular topic and to identify research gaps for developing future research (Evangelista, Santoro, \& Thomas, 2018). It also consists of a well-defined methodical process and an analytical framework rendering the search criteria and search process transparent (Koch, Kabisch, \& Krellenberg, 2017) and easily replicable. In the context of sustainability assessment, Cohen (2017) and Silva, Beske-Janssen, and Schaltegger (2017) represent recent examples of studies that have adopted an SLR approach to provide an overview of the state or best practice of urban sustainability assessment and measurement. The authors have adopted the SLR approach as outlined by Tranfield, Denyer, and Smart (2003), which has been applied by Silva, Beske-Janssen, and Schaltegger (2017) and Evangelista, Santoro, and Thomas (2018) in their systematic review studies. As outlined by Tranfield, Denyer, and Smart (2003) systematic reviews differ from conventional narrative reviews "by adopting a replicable, scientific and transparent process, in other words, a detailed technology, that aims to minimize bias through exhaustive literature searches of published and unpublished studies and by providing an audit trail of the reviewer's decisions, procedures, and conclusions". The approach consisted of five methodological stages including (1) identification of studies, (2) study selection, (3) study quality assessment, (4) data extraction, and (5) data 
synthesis and reporting (Silva, Beske-Janssen, \& Schaltegger, 2017) (see Figure 2).

The content analysis conducted in the SLR is keyword-driven and is based on the study of Cohen (2017). In most of the SLR studies, keyword extraction is done manually which is a tedious, expensive and a poorly scalable task prone to human bias and random error. In order to ameliorate this, the authors felt the need to develop a software that uses an unsupervised machine learning algorithm called RAKE to automatically extract keywords from the systematically selected articles and gives a quick composite picture of the state of sustainability assessment literature. The software is written in Python which is one of the most globally popular programming languages. The entire source code of the project is freely available on GitHub ${ }^{1}$. Keywords (more generally, keyphrases) represent the essential content of a document in a condensed form. Automatic Keyphrase Extraction (AKE) refers to the task of "automatic selection of important and topical phrases from the body of a document” (Turney, 2000).

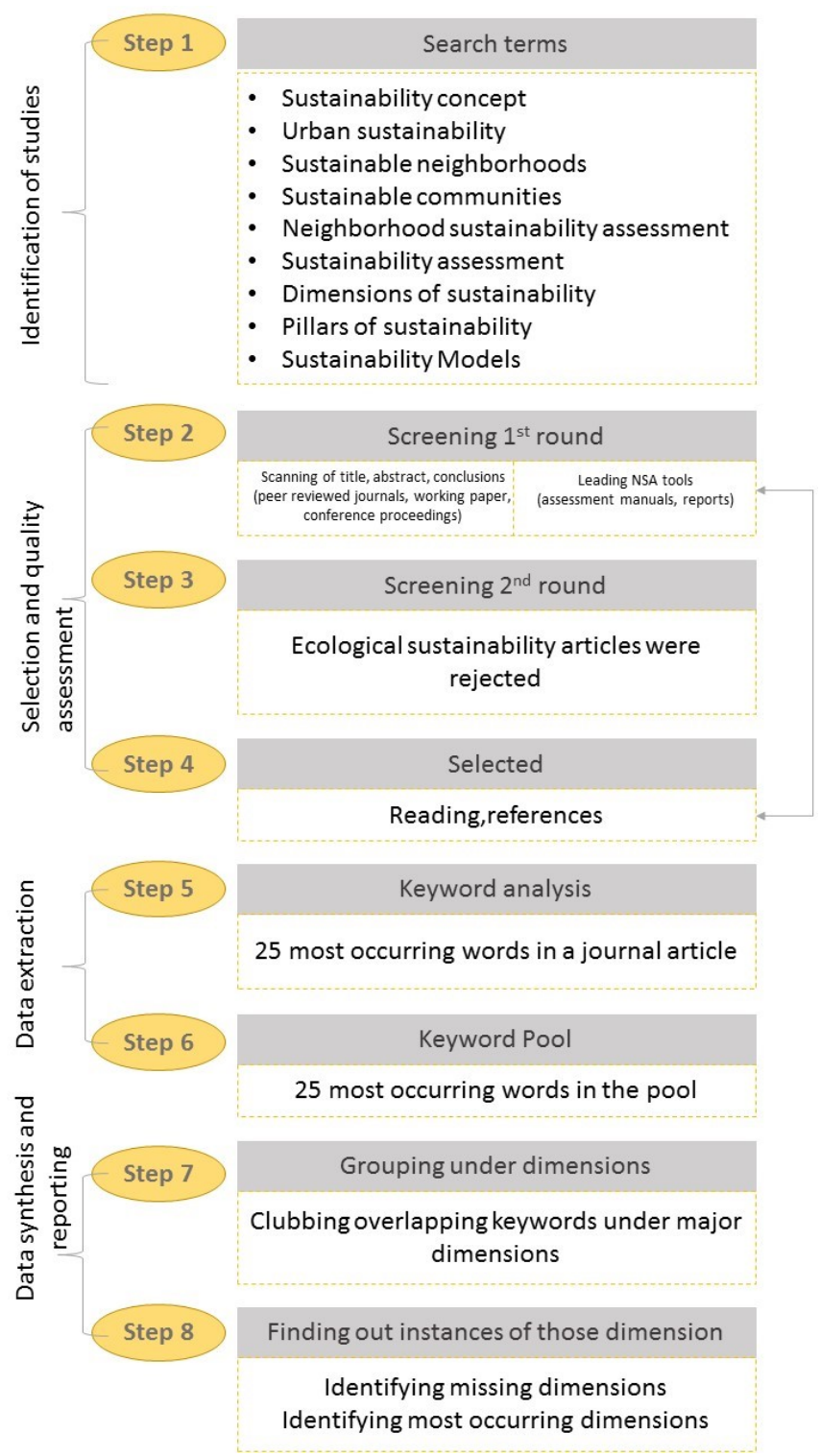

Figure 3. Showing the SLR methodology

\footnotetext{
${ }^{1}$ https://github.com/Santara/autoSLR
} 
Keyphrases enable fast and accurate search for particular characteristics within huge volumes of text, required for tasks like text-summarization (Zhang, Heywood, \& Milios, 2004), text categorization (Hulth \& Megyesi, 2006), opinion mining (Berend, 2011) and document indexing (Gutwin et al., 1999). The classic approach to AKE consists of two steps: (1) using some heuristics (e.g. removal of stop-words (Leskovec, Rajaraman, \& Ullman, 2014), prioritizing certain parts of speech, searching for nounphrases with pre-defined lexico-syntactic patterns, etc.) to generate a list of candidate words/phrases; (2) determining which of the candidates qualify as keyphrases using supervised and unsupervised techniques (Hasan \& Ng, 2014).

This study uses the Rapid Automatic Keyword Extraction (RAKE) algorithm (Rose et al., 2010) which is an efficient, unsupervised, domainindependent and language-independent unsupervised machine learningbased method for extracting keywords from documents. The input to RAKE consists of a list of stop-words (stop-list), a set of phrase delimiters and a set of word delimiters in addition to the document to be searched. Candidate keyphrases are generated by first splitting the document into words using the word-delimiters and then splitting sequences of contiguous words at phrase delimiters and stop-word positions. To find key phrases with interior stopwords (e.g. axis of evil), RAKE looks for pairs of adjoining keywords that have appeared at least twice in the document in the same order. Next, a graph of phrase co-occurrences is generated for the candidate key phrases and scores are generated based on the degree and frequency of the vertices in the graph. Finally, the top $T$ scoring candidates are chosen as key phrases for the document.

\section{DETAILED METHODOLOGY AND RESULTS}

This section describes the detailed methodology of the SLR. The foremost aim of the search process is to identify the missing dimensions in sustainability research. The motivation of the SLR is to conduct a datadriven validation of the observations made by the authors regarding missing and neglected dimensions in the study of sustainability of NLUC's.

\subsection{Search strategy}

The search process is based on sustainability assessment in general and is mainly focused on the context of neighbourhood sustainability assessment and measurement. The authors have searched articles from digital libraries including academic journals, conference proceedings, government publications, and reports produced by leading practitioners. These included Google search engine, Google scholar, Springer link, Science direct / Scopus, MDPI (especially Sustainability journal), Taylor and Francis, Wiley Inter-science, and Web of Science U-M library. An initial set of relevant search terms was identified based on previous research and terminologies used in the previous section of this study, the aim of the study, and authors' knowledge on the topic.

The search strategy started with the search terms of:

1. Sustainability concept

2. Urban sustainability

3. Sustainable communities/neighbourhoods 
4. Sustainability assessment

5. Neighbourhood sustainability assessment

6. Dimensions of sustainability

7. Pillars of sustainability

8. Sustainability models

Besides the peer-reviewed journal articles, a grey literature search was also performed with the term 'sustainability assessment', 'sustainability models', 'sustainable communities', etc. using Google's search engines. Also, leading neighbourhood sustainability assessment manuals were identified and included in the selected list of articles.

\subsection{Selection of articles}

Over 120 articles were selected in the first round of selection based on the search terminologies. The authors have adopted a three-step screening process. Firstly, a qualitative content analysis was conducted. The articles were manually selected by the rapid reading of their title, abstract, and conclusion. Articles which had ecologically biased studies were rejected since the major aim of this study was to identify the missing dimensions in sustainability assessment and to devise a comprehensive sustainability framework. The time span of these articles is from 1998-2018, articles prior to 1998 were also rejected. All selected articles were classified along with the following categories: 1) source, 2) journal of publication, 3) topic (Koch, Kabisch, \& Krellenberg, 2017), 4) spatial scale, and 5) location (Cohen, 2017). The references of the selected articles were also checked and the relevant ones were selected for a review process. By this process, 65 articles were processed. Out of the 65 articles, 40 were journal articles and their source has been included in Table 1 (see Section 3.5). The remaining 25 articles were mostly grey literature comprised of reports, conference papers, working papers and assessment manuals - their sources have been included in Table 2 (see Section 3.5). The twelve most studied NSA tools from different regions in the world were selected for the SLR. Their online available assessment manuals were included in the selected list of articles. These include the assessment manuals of LEED, BREAM, Green Township, BEAM, BCA Green Mark, Earth Craft Communities, Green Mark District, EcoCity, and STAR Community and Neighbourhood Sustainability Framework New Zealand. ${ }^{2}$

\subsection{Keyword extraction}

With a view to ameliorate human bias and random error, the authors used the Rapid Automatic Keyword Extraction (RAKE) algorithm (Rose et al., 2010), to extract the 25 most frequently occurring keywords in each article. In this study, only single-word keywords were chosen. If RAKE output 25 or fewer keywords for an article, all of them were chosen. Otherwise, the 25 most occurring keywords were selected. In this way, a pool of 1,452 keywords was generated.

\footnotetext{
2 The list of articles is given in

https://drive.google.com/file/d/1TiSPGckRyCq0jHNGuFDZAtbpO2C4gve2/view?usp=sharin 


\subsection{Grouping under dimensions}

Sustainable development objectives are conventionally defined by economic, social and environmental dimensions (Spangenberg, 2002). However, some recent research has taken serious note of institutional /cultural dimensions as other pillars of sustainability which need to be complemented by core institutional objectives including a mechanism for management and implementation in order to achieve justice and encourage public participation (Spangenberg, 2002). In 1996, Stephen Viedarman defined sustainability in terms of shared capital and constant capital, and introduced the concept of cultural capital which is essential in the context of sustainable communities (Mazmanian \& Kraft, 2009). Traditionally, cultural aspects were viewed as important components of the social dimension, but currently, culture is being recognised as a separate dimension of sustainability (Duxbury, Gillette, \& Pepper, 2007). Jon Hawkes, a cultural analyst in 2001, raised the need for incorporating a cultural perspective in public planning and policy for interlinking environmental responsibility, economic health, social equity and cultural vitality, and justified culture as the fourth pillar of sustainability (Duxbury, Gillette, \& Pepper, 2007). Despite many efforts by sociologists, planners, social geographers and political ecologists to date, the social pillar continues to occupy a lower status in the holy trinity of sustainable development (Parra \& Moulaert, 2010). The authors opine that a strong positive integration between the three pillars of sustainability is needed for effective triggering of sustainable development. In the last decade, the prism of sustainability, as shown in Figure 3, adapted by a German research institute, the Wuppertal Institute, has proposed four dimensions, namely economy, environment, social and institution (Keiner, 2005).

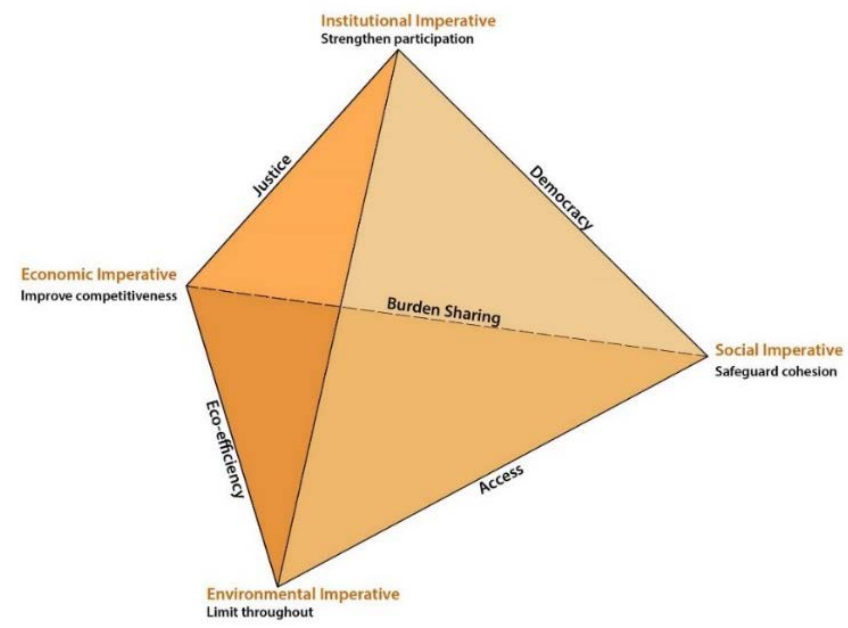

Figure 4. Showing the prism of sustainability

After the keywords were extracted, the stemmed keywords, such as with its prefix, suffix and pluralization, were clubbed together. After selecting the 25 most occurring keywords, the authors adopted the most conventional structure of grouping keywords under the traditional pillars of sustainability (Refer Figure 4). A lot of studies have considered a four pillars' view: environmental, economic, social and cultural, while some other studies have considered institutional as the fourth dimension. Only 15 studies out of 65 
have mentioned all the five dimensions, but those that have dealt with some aspects of cultural and institutional dimensions have not considered them in a major way.

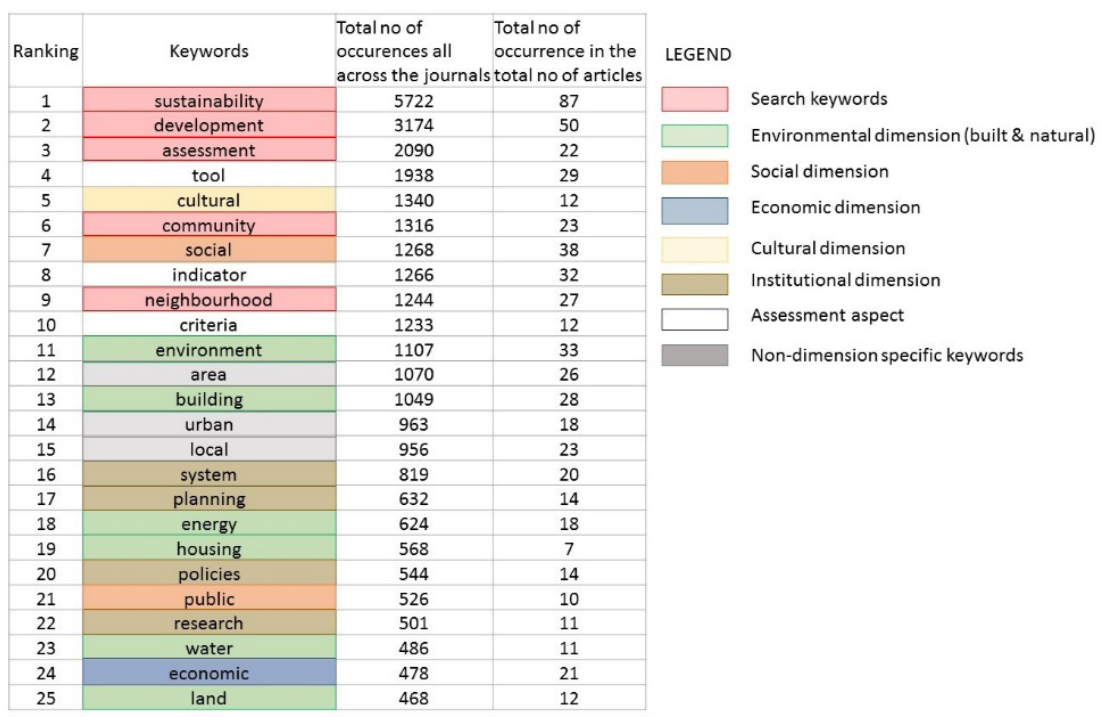

Figure 5. Showing the grouping of the keywords under each dimension

To the best of the authors' knowledge, the five dimensions have never been jointly considered in the discussion on neighbourhood sustainability assessment. The authors investigated the importance of studying all five dimensions - environment, economic, social, cultural and institutional - for assessing the sustainability of NLUCs.

A given article was considered to have studied a given dimension if any of the keywords corresponding to that dimension had appeared more than a threshold $t$ number of times. The authors chose $t=5$ after an empirical analysis - described in the next section - to maximise agreeability with a human expert. The results are shown in the following bar graphs. The results of the previous step were used to categorize the articles as to whether they studied three, four or five dimensions as described in Table 1. The number of articles in each category has been presented in the following bar graph.

Number of Articles Studying Specific Dimensions

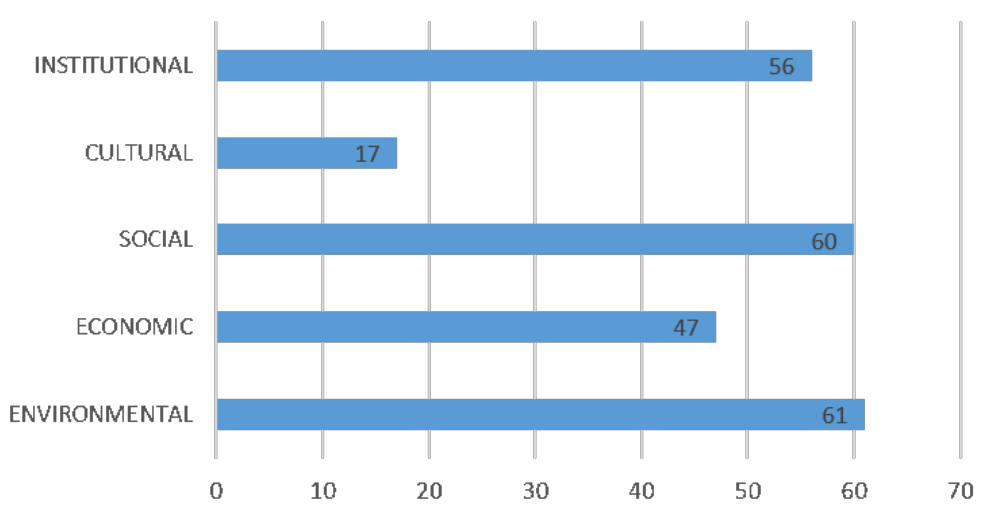

Figure 6. Bar graph showing the number of articles studying specific dimensions 


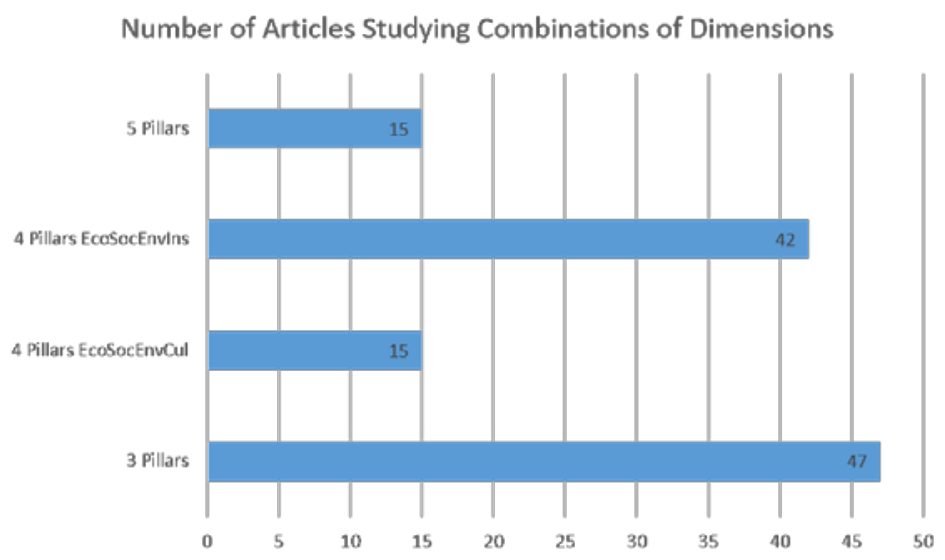

Figure 7. Bar graph showing the number of articles studying combination of dimensions

The authors have found that more than 95\% of articles have talked about environment, economic and social dimensions in their sustainability assessment frameworks. Only 15 out of 65 articles have considered cultural aspects along with the aforementioned traditional three pillars. Some of the articles have considered cultural as the fourth pillar of sustainability. About 42 articles have taken into consideration aspects like governance, urban planning, public or research within the sustainability frameworks, which the authors have grouped together under institutional dimensions in this study. Only 15 articles out of 65 have talked about environment, economic, social, cultural and institutional aspects in their sustainability frameworks, but none of them have considered all the five dimensions together in a comprehensive holistic framework.

\subsection{Choice of the threshold for classification}

This section describes a data-driven study that was conducted to choose the value of threshold $t$ on the minimum number of occurrences of a keyword needed for the presence of a given dimension. The goal was to maximize agreeability with human experts. The authors manually read all the selected articles and labelled them as per the number of dimensions discussed (as described in Table 1).

Table 1. Showing the combination of the pillars

\begin{tabular}{|l|c|r|r|r|c|}
\hline & Environmental & Economic & Social & Cultural & Institutional \\
\hline Three Pillars & $\checkmark$ & $\checkmark$ & $\checkmark$ & & \\
\hline $\begin{array}{l}\text { Four Pillars } \\
\text { EcoSocEnvCul }\end{array}$ & $\checkmark$ & $\checkmark$ & $\checkmark$ & $\checkmark$ & \\
\hline $\begin{array}{l}\text { Four Pillars } \\
\text { EcoSocEnvIns }\end{array}$ & $\checkmark$ & $\checkmark$ & $\checkmark$ & & $\checkmark$ \\
\hline Five Pillars & $\checkmark$ & $\checkmark$ & $\checkmark$ & $\checkmark$ & $\checkmark$ \\
\hline
\end{tabular}

The expert labels were compared with the output of the automated classifier of Section 3.4 and the number of true positives (TP), true negatives (TN), false positives (FP) and false negatives (FN), defined in Table 2, were counted. 
Table 2. Showing the actual and prediction composite matrix

Prediction

\begin{tabular}{|c|c|c|}
\hline & FALSE & TRUE \\
\hline FALSE & $\mathrm{TN}$ & FP \\
\hline TRUE & FN & $\mathrm{TP}$ \\
\hline
\end{tabular}

Sensitivity and specificity are statistics that are used to measure the performance of a binary classification (Altman \& Bland, 1994). Sensitivity measures the proportion of the actual positives which are correctly identified. Specificity measures the proportion of the actual negatives which are correctly identified (Refer Formula 1.1). Both sensitivity and specificity are real numbers between 0 and 1 , the higher the more sensitive or specific.

$$
\text { Sensitivity }=\frac{T P}{T P+F N}, \text { Specificity }=\frac{T N}{F P+T N}
$$

Sensitivity and specificity for different values of threshold, $t$, between 0 10 were plotted and their behaviours were studied for the proposed automated classifier. The authors have also found the operating point for each of the pillars. The operating point implies that for a particular threshold both the sensitivity and specificity values are high.
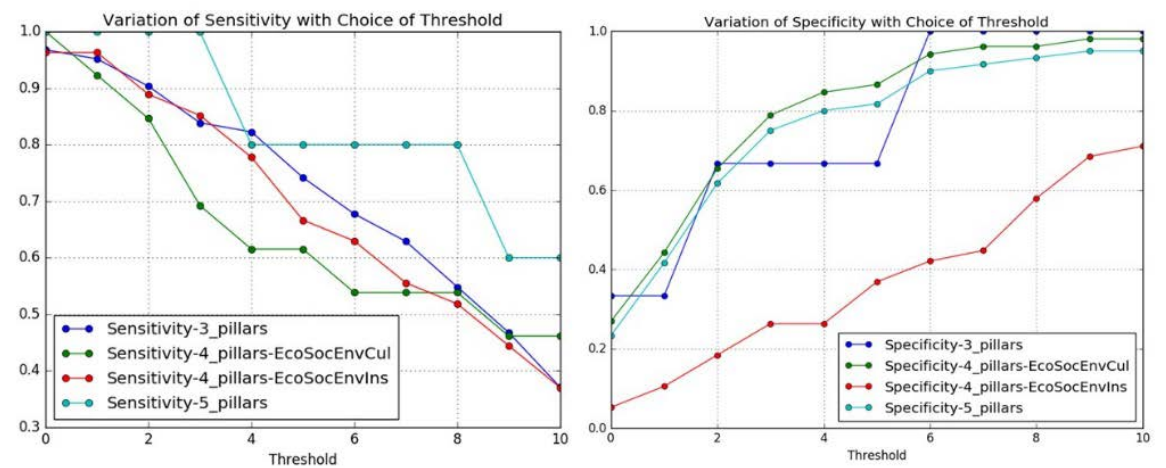

Figure 8. Variation of sensitivity and specificity of the classifier with the choice of the minimum number of occurrences of a characteristic keyword needed for the presence of a particular dimension

Sensitivity and specificity for thresholds ranging from 0-10 were plotted and their behaviours were also studied. The authors have also found the operating point for each of the pillars. The operating point implies that for a particular threshold both the sensitivity and specificity values are high. 

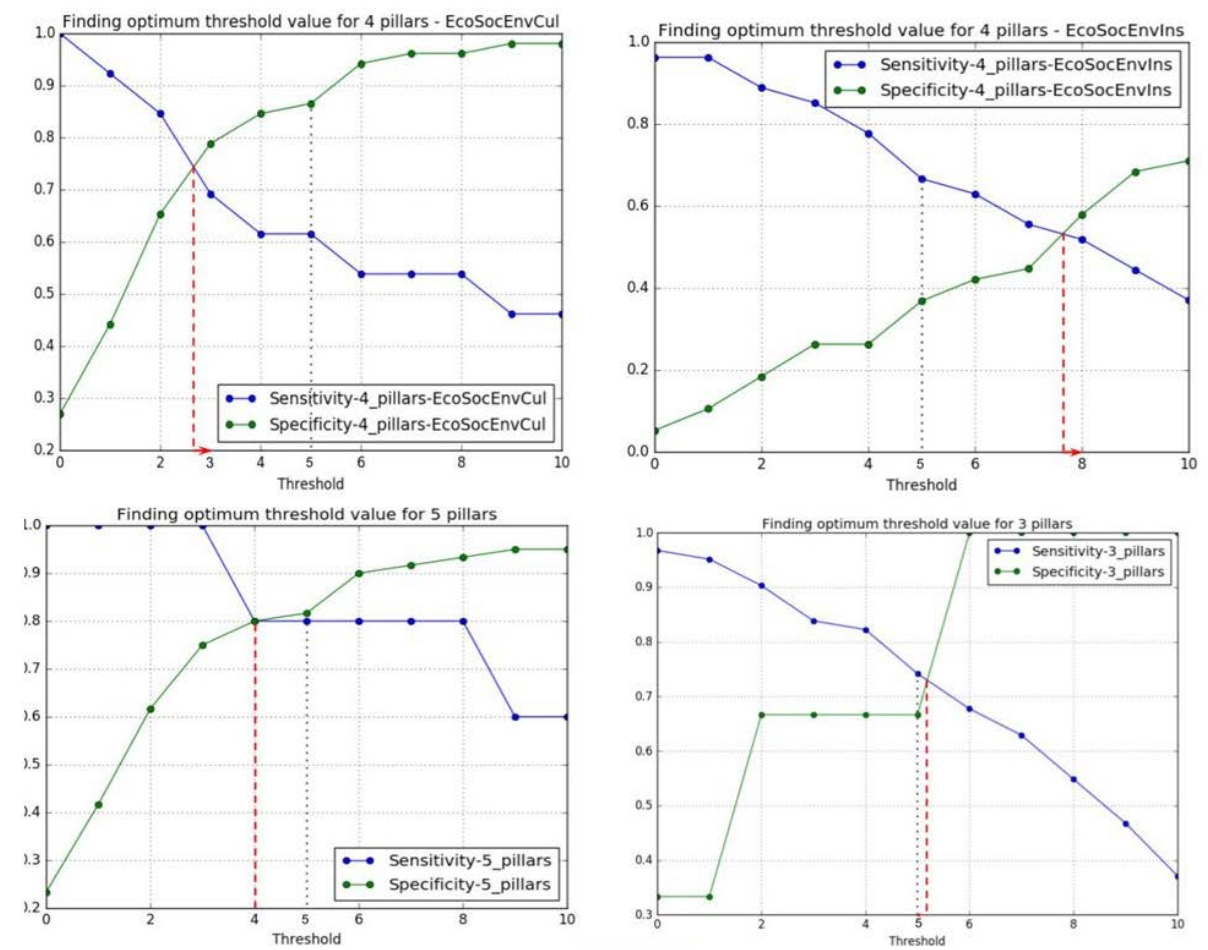

Figure 9. Showing the operating point for the automated dimension classifier

It has been observed that the chosen value of threshold equal to 5 is close to the optimal choice for three or five pillars and the average value of the optimal threshold for the two variants of four pillars. Hence the choice is legitimate (Refer Table 3).

Table 3. Showing the loss in sensitivity and specificity due to the chosen value of threshold equal to 5

\begin{tabular}{|c|c|c|c|}
\hline $\begin{array}{c}\text { Pillar } \\
\text { Combinations }\end{array}$ & $\begin{array}{c}\text { Threshold } \\
\text { corresponding to } \\
\text { Optimal } \\
\text { Operating-Point }\end{array}$ & $\begin{array}{c}\text { Loss in } \\
\text { sensitivity due to } \\
\text { chosen value of } \\
\text { threshold equal to 5 }\end{array}$ & $\begin{array}{c}\text { Loss of } \\
\text { specificity due to } \\
\text { chosen value of } \\
\text { threshold equal } \\
\text { to 5 }\end{array}$ \\
\hline Three Pillars & 5 & $0 \%$ & $0 \%$ \\
\hline $\begin{array}{c}\text { Four Pillars } \\
\text { EcoSocEnvCul Pillars }\end{array}$ & 3 & $10.1 \%$ & $-11.5 \%$ \\
\hline $\begin{array}{c}\text { Four EcoSocEnvIns } \\
\text { Five Pillars }\end{array}$ & 8 & $-26.9 \%$ & $37.9 \%$ \\
\hline
\end{tabular}

Limitations of the proposed method:

- RAKE does not stem or lemmatise the keywords. Stemming and lemmatisation are methods used in Natural Language Processing to reduce inflectional forms and derivationally related forms of a word to a common base form in order to consider the occurrences of the same word in different inflected/derived forms in different documents/contexts with equal importance (For example: am, are, is $\rightarrow$ be; car, cars, car's, cars' $\rightarrow$ car) (Manning, Raghavan, \& Schütze, 2008). Stemming is the simple process of pruning words 
from the end towards finding the base form. Lemmatisation takes a more principled approach and uses morphological analysis and a lexicon to arrive at the true base form of the word (called "lemma") by identifying and removing just the inflectional endings (Manning, Raghavan, \& Schütze, 2008). The authors ameliorated this by manually clubbing together the occurrences of different inflected/derived forms of the same keyword. Some of the pdf files are protected and hence keywords were not extracted since the code was unable to process those files. The authors had to reject those files.

\section{$4 \quad$ DISCUSSION}

This paper has proposed a theoretical pentagram model for sustainable communities which has included cultural and institutional dimensions along with the existing three fundamental pillars of sustainability. To date, there is no study which has actually identified the missing dimensions of sustainability and incorporated them along with the conventional dimensions to create a new holistic model. In this model all the five dimensions have been considered exclusively within the context of NLUCs. Some of the studies have identified the need for the inclusion of a cultural and institutional dimension in sustainability models, but to date they haven't been considered seriously in the existing models. An institutional aspect will be elemental in implementing policies and a cultural aspect will be elemental in enhancing community engagement and participation, making it more location specific. This will provide a platform for integration of the intrinsic cultural characteristics of the community within the public realm (Hawkes, 2001). Within the institutional framework, the cultural framework will operate in parallel with the social, economic and environmental frameworks. This is necessary for achieving a healthy society (Hawkes, 2001) resulting in sustainable communities.

The pentagram as shown in Figure 9 works based on the interlinking approach of the prism of sustainability (Refer Figure 3) and represents interconnectedness, transdisciplinarity, and coherence among the different dimensions. This model proposes to deal with five dimensions of sustainability, namely economic, social, environmental, cultural and institutional. The pentagram of sustainability states the importance of interaction between each dimension as demonstrated in Figure 7. This model will be a significant contribution to the body of contemporary research in this field for sustainability assessment of NLUCs. This model provides versatility by incorporating the regional specific cultural and political context by exclusively treating culture and institution as specific dimensions unlike underlying components as evident in existing sustainability models. Each one of the five dimensions will have an overarching component of innovation in order to accommodate the impact on them due to technological advancements and future trends which have made the model even more flexible. 


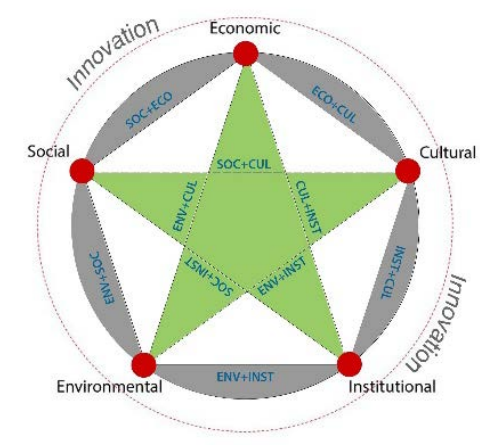

Figure 10. Illustrating the five dimensions along with interlinkages

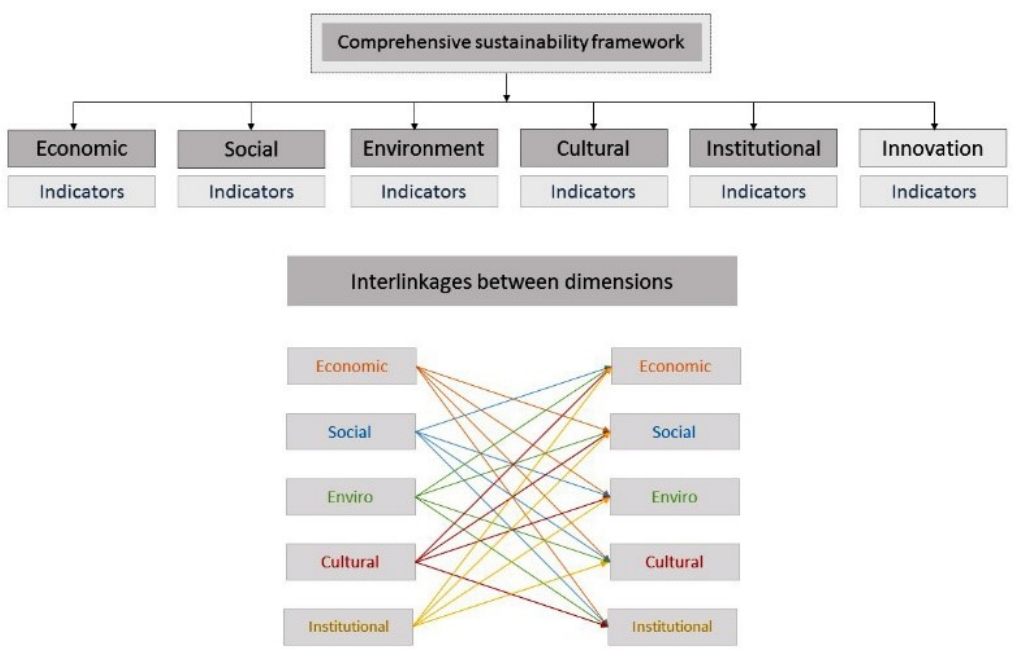

Figure 11. Illustrating the dimensional framework for assigning indicators

\section{$5 \quad$ CONCLUSIONS}

This paper conducted an exhaustive, data driven SLR on the existing literature on neighbourhood sustainability assessment to identify the missing and neglected sustainability dimensions. The paper confers that the triple bottom line and the Wuppertal sustainability models lack full integration of all the different dimensions discussed and also consideration of the interconnectedness amongst them. The importance of the cultural and institutional dimensions in developing a holistic framework for achieving sustainability of the communities has been well established by the authors in this paper. The inclusion of cultural and institutional dimensions is strongly recommended for a more region-specific approach towards implementation of policies and the state agenda without compromising the environmental, economic, social and cultural aspects of the community. The pentagram model works based on the interlinkage approach of the prism of sustainability and represents interconnectedness, transdisciplinarity, and coherence among the different dimensions. The outcome of this model will provide a basis for a framework structure within which indicators will be assigned for assessing the sustainability of the NLUCs. The relevance of 
each of the linkages can be exclusively explored and pursued by future researchers in this field.

The semi-automated SLR methodology devised by the authors will expedite the literature review process. The SLR has led the authors to outline a holistic comprehensive dimensional framework for assessing sustainability of NLUCs in India. This dimensional framework will benefit the field of neighbourhood sustainability assessment. The framework can also be used for assessing the effectiveness of the existing NSA tools with the help of empirical assessment studies. The existing indicators of the NSA tools can be assigned under the corresponding dimensions from which the strength and weakness of each tool can be known, and which dimension is prioritised by which tool and which are the neglected dimensions can be determined. This would help researchers to understand the persisting research gaps in the field of neighbourhood sustainability assessment.

\section{ACKNOWLEDGEMENTS}

We acknowledge the Department of Architecture and Regional Planning, Indian Institute of Technology Kharagpur for financially supporting this study.

\section{REFERENCES}

Altman, D. G., \& Bland, J. M. (1994). "Diagnostic Tests. 1: Sensitivity and Specificity". BMJ: British Medical Journal, 308(6943), 1552.

Barrett, C. B., \& Grizzle, R. (1999). "A Holistic Approach to Sustainability Based on Pluralism Stewardship". Environmental Ethics, 21(1), 23-42.

Berardi, U. (2013). "Sustainability Assessment of Urban Communities through Rating Systems". Environment, development sustainability, 15(6), 1573-1591.

Berend, G. (2011). "Opinion Expression Mining by Exploiting Keyphrase Extraction". Proceedings of 5 th International Joint Conference on Natural Language Processing, Thailand.

Bhatt, R., Macwan, J., \& Bhatt, D. (2012). "Sustainable Building Assessment Tool: Indian Leading Architects' Perceptions and Preferences". Journal of The Institution of Engineers : Series A, 93(4), 259-270.

Bijoux, D., \& Pathway, B. (2012). "A Neighbourhood Sustainability Framework for New Zealand: Beacon's Research and Tools".

Blum, A. (2007). "Hqe2r-Research and Demonstration for Assessing Sustainable Neighborhood Development". Sustainable urban development, 2, 412-428.

Bond, A., Morrison-Saunders, A., \& Pope, J. (2012). "Sustainability Assessment: The State of the Art". Impact Assessment Project Appraisal, 30(1), 53-62.

Cheshire, P. C. (2007). Segregated Neighbourhoods and Mixed Communities: A Critical Analysis. the UK: Joseph Rowntree Foundation.

Cohen, M. (2017). "A Systematic Review of Urban Sustainability Assessment Literature". Sustainability, 9(11), 2048.

Dalal-Clayton, B., \& Sadler, B. (2014). Sustainability Appraisal: A Sourcebook and Reference Guide to International Experience. the UK: Routledge.

Duxbury, N., Gillette, E., \& Pepper, K. (2007). "Exploring the Cultural Dimensions of Sustainability". Creative City News: Special Edition, 4, 1-4.

Elkington, J. (1997). Cannibals with Forks: The Triple Bottom Line of Twenty-First Century Business. Capstone. the UK: Oxford.

Evangelista, P., Santoro, L., \& Thomas, A. (2018). "Environmental Sustainability in ThirdParty Logistics Service Providers: A Systematic Literature Review from 2000-2016". Sustainability, 10(5), 1627.

Geis, D., \& Kutzmark, T. (1998). "Developing Sustainable Communities the Future Is Now". Retrieved from http://www.freshstart.ncat.org/articles/future.htm. 
Gibson, R. B. (2006). "Sustainability Assessment: Basic Components of a Practical Approach". Impact Assessment Project Appraisal, 24(3), 170-182.

Gutwin, C., Paynter, G., Witten, I., Nevill-Manning, C., \& Frank, E. (1999). "Improving Browsing in Digital Libraries with Keyphrase Indexes". Decision Support Systems, 27(12), 81-104.

Haapio, A. (2012). "Towards Sustainable Urban Communities". Environmental Impact Assessment Review, 32(1), 165-169.

Hacking, T., \& Guthrie, P. (2008). "A Framework for Clarifying the Meaning of Triple Bottom-Line, Integrated, and Sustainability Assessment". Environmental Impact Assessment Review, 28(2-3), 73-89.

Hasan, K. S., \& Ng, V. (2014). "Automatic Keyphrase Extraction: A Survey of the State of the Art". Proceedings of Proceedings of the 52nd Annual Meeting of the Association for Computational Linguistics (Volume 1: Long Papers), pp. 1262-1273.

Hassan, A. M., \& Lee, H. (2015). "The Paradox of the Sustainable City: Definitions and Examples". Environment, development sustainability, 17(6), 1267-1285.

Hawkes, J. (2001). The Fourth Pillar of Sustainability: Culture's Essential Role in Public Planning. Common Ground.

Hopwood, B., Mellor, M., \& O'Brien, G. (2005). "Sustainable Development: Mapping Different Approaches". Sustainable development, 13(1), 38-52.

Huang, L., Wu, J., \& Yan, L. (2015). "Defining and Measuring Urban Sustainability: A Review of Indicators". Landscape ecology, 30(7), 1175-1193.

Hulth, A., \& Megyesi, B. B. (2006). "A Study on Automatically Extracted Keywords in Text Categorization". Proceedings of 21st International Conference on Computational Linguistics and the 44th annual meeting of the Association for Computational Linguistics, pp. 537-544.

Jabareen, Y. (2009). "Building a Conceptual Framework: Philosophy, Definitions, and Procedure". International journal of qualitative methods, 8(4), 49-62.

Keiner, M. (2005). "History, Definition (S) and Models of Sustainable Development". ETH Zurich.

Koch, F., Kabisch, S., \& Krellenberg, K. (2017). "A Transformative Turn Towards Sustainability in the Context of Urban-Related Studies? A Systematic Review from 1957 to 2016". Sustainability, 10(1), 1-17.

Komeily, A., \& Srinivasan, R. S. (2015). "A Need for Balanced Approach to Neighborhood Sustainability Assessments: A Critical Review and Analysis". Sustainable Cities Society, 18, 32-43.

Komeily, A., \& Srinivasan, R. S. (2016). "What Is Neighborhood Context and Why Does It Matter in Sustainability Assessment?". Procedia Engineering, 145, 876-883.

Kramers, A., Wangel, J., Johansson, S., Höjer, M., Finnveden, G., \& Brandt, N. (2013). "Towards a Comprehensive System of Methodological Considerations for Cities' Climate Targets". Energy policy, 62, 1276-1287.

Kyvelou, S., Sinou, M., Baer, I., \& Papadopoulos, T. (2012). "Developing a South-European Eco-Quarter Design and Assessment Tool Based on the Concept of Territorial Capital". Sustainable Development-Authoritative and Leading Edge Content for Environmental Management: IntechOpen.

Leskovec, J., Rajaraman, A., \& Ullman, J. (2014). "Mining of Massive Datasets: (2014)". Cambridge: Cambridge University Press.

Lin, K. W., \& Shih, C. M. (2016). "The Comparative Analysis of Neighborhood Sustainability Assessment Tool". Environment Planning B: Urban Analytics City Science, 45(1), 90-105.

Manning, C. D., Raghavan, P., \& Schütze, H. (2008). Introduction to Information Retrieval. (Vol. 39). the UK: Cambridge university press.

Marshall, J. D., \& Toffel, M. W. (2005). "Framing the Elusive Concept of Sustainability: A Sustainability Hierarchy". (0013-936X), ACS Publications.

Mazmanian, D. A., \& Kraft, M. E. (2009). Toward Sustainable Communities: Transition and Transformations in Environmental Policy. MIT Press.

Panda, S., Chakraborty, M., \& Misra, S. (2016). "Assessment of Social Sustainable Development in Urban India by a Composite Index". International Journal of Sustainable Built Environment, 5(2), 435-450.

Parra, C., \& Moulaert, F. (2010). "Why Sustainability Is So Fragilely "Social”". Strategic spatial projects: Catalysts for change, 163-173.

Pope, J., Bond, A., Huge, J., \& Morrison-Saunders, A. (2017). "Reconceptualising Sustainability Assessment". Environmental Impact Assessment Review, 62, 205-215.

Rose, S., Engel, D., Cramer, N., \& Cowley, W. (2010). "Automatic Keyword Extraction from Individual Documents". Text mining: applications theory, 1, 1-20.

Searfoss, L. (2011). "Local Perspectives on Hud's Neighborhood Stabilization Program". 
MUP Capstone.

Sharifi, A., \& Murayama, A. (2013). "A Critical Review of Seven Selected Neighborhood Sustainability Assessment Tools". Environmental Impact Assessment Review, 38, 73-87.

Sharifi, A., \& Murayama, A. (2014). "Neighborhood Sustainability Assessment in Action: Cross-Evaluation of Three Assessment Systems and Their Cases from the Us, the Uk, and Japan". Building Environment, 72, 243-258.

Sharifi, A., \& Murayama, A. (2015). "Viability of Using Global Standards for Neighbourhood Sustainability Assessment: Insights from a Comparative Case Study". Journal of Environmental Planning Management, 58(1), 1-23.

Silva, S. L., Beske-Janssen, P., \& Schaltegger, S. (2017). "Measuring Positive Sustainability. A Systematic Literature Review". Business Ethics, 133(2), 193-221.

Spangenberg, J. H. (2002). "Institutional Sustainability Indicators: An Analysis of the Institutions in Agenda 21 and a Draft Set of Indicators for Monitoring Their Effectivity". Sustainable development, 10(2), 103-115.

Tanguay, G. A., Rajaonson, J., Lefebvre, J.-F., \& Lanoie, P. (2010). "Measuring the Sustainability of Cities: An Analysis of the Use of Local Indicators". Ecological Indicators, 10(2), 407-418.

Tranfield, D., Denyer, D., \& Smart, P. (2003). "Towards a Methodology for Developing Evidence-Informed Management Knowledge by Means of Systematic Review". British journal of management, 14(3), 207-222.

Turney, P. D. (2000). "Learning Algorithms for Keyphrase Extraction". Information retrieval, 2(4), 303-336.

Wackernagel, M., \& Rees, W. (1998). Our Ecological Footprint: Reducing Human Impact on the Earth. (Vol. 9) New society publishers.

Wang, Y. (2014). "The Framework of Social Sustainability for Chinese Communities: Revelation from Western Experiences". International review for spatial planning sustainable development, 2(3), 4-17.

Zhang, Y., Heywood, N., \& Milios, E. (2004). "World Wide Web Site Summarization Web Intelligence and Agent Systems". Technical Report, CS-2002-8. 